\title{
Directed anomalous diffusion without a biased field: A ratchet accelerator
}

\author{
Jiangbin Gong* and Paul Brumer \\ Chemical Physics Theory Group, Department of Chemistry, University of Toronto, Toronto, Canada M5S 3 H6
}

(Received 20 January 2004; published 8 July 2004)

\begin{abstract}
Directed classical current that increases linearly with time without using a biased external field is obtained in a simple model Hamiltonian system derived from a modified kicked rotor model, by breaking the spatial symmetry of the transporting regular islands in the classical phase space. A parallel study of the corresponding quantum dynamics suggests that although quantum coherence effects suppress the directed current and can even induce a current reversal, directed quantum current that increases linearly with time can nonetheless be realized in a quantum system that is far from the classical limit.
\end{abstract}

DOI: 10.1103/PhysRevE.70.016202

PACS number(s): 05.45.Mt, 32.80.Qk, 05.60.-k

\section{INTRODUCTION}

Ratchet effects, i.e., directed transport without a biased field due to a broken spatial-temporal symmetry, are of great interest to the understanding of molecular motors in biological systems and have been under intense investigation in a variety of simple model systems [1,2]. Early studies focused on the role of external noise, which was then replaced by deterministic chaotic dynamics with dissipation [3-6]. Recently, it was shown that even purely Hamiltonian dynamics with both regular and chaotic phase space structures [7-10] or with complete chaos [6,11-13] is capable of generating ratchet effects. As such, there has been a great interest in studying simple model Hamiltonian systems in an effort to observe and understand ratchet effects.

The kicked rotor (KR) model [14] and its variants are ideal systems for such studies. For example, delta-kicked systems with an alternating kicking period and an asymmetric potential $[9,11,12]$, or with multiple external fields [10], have been considered in connection with ratchet effects. These studies are stimulating since the classical dynamics of the KR (i.e., the standard map) and the associated quantum dynamics have long served as paradigms for classical and quantum chaos [14]. Indeed, the KR has been realized in several atom optics experimental groups [15], and is also of considerable interest in other fields such as condensed matter physics [16,17], molecular physics [18-21], and quantum information science $[22,23]$.

Although not often thought of as such, the basic idea that underlies the above-mentioned studies of ratchet effects in the KR, i.e., to manipulate symmetry properties to induce a directed current, is not new from a coherent control perspective. Indeed, 15 years ago Kurizki, Shapiro, and Brumer [24] proposed a simple coherent control scenario that can generate photocurrents in semiconductors without a biased voltage. This proposal and its various extensions [25,26] have been experimentally realized [27-29].

In this paper we introduce a totally different type of Hamiltonian ratchet effect in a simple $\delta$-function kicked

\footnotetext{
*Current address: Department of Chemistry and The James Franck Institute, University of Chicago, Chicago, IL 60637.
}

model system, which we call a "ratchet accelerator." Without a net external force (i.e., the external force averaged over space is zero), the ratchet accelerator can generate directed classical anomalous transport, with the net current (defined below) that accelerates linearly with time. This is made possible by taking advantage of the large transporting islands in the classical phase space of a modified kicked rotor system recently proposed by Gong, Wörner, and Brumer [30,31], in conjunction with a second kicking field that breaks the spatial symmetry. The corresponding quantum dynamics is also studied, shedding considerable light on quantum-classical differences and correspondence in the presence of anomalous diffusion. For example, we show that due to the tunneling between a chaotic sea and a relatively large regular region of the classical phase space, the quantum directed current can be in the opposite direction of the classical directed current.

This paper is organized as follows. In Sec. II we describe the background and the motivation of our model as a ratchet accelerator. The classical results of directed anomalous diffusion are presented in Sec III, followed by the analogous quantum results in Sec. IV. Concluding remarks are given in Sec. V.

\section{MODEL SYSTEM}

Consider the following model Hamiltonian:

$$
\begin{aligned}
H(p, x, t)= & p^{2} / 2 m+\lambda_{1} \cos \left(x / x_{0}\right) \sum_{n} f(n) \delta(t / T-n) \\
& -\lambda_{2} \sin \left(2 x / x_{0}\right) \sum_{n} \delta(t / T-n)
\end{aligned}
$$

where $p$ is the momentum, $m$ is the particle mass, $x$ is the conjugate position, $\lambda_{1}$ and $\lambda_{2}$ are the strength of the first and second kicking fields, $T$ is the time interval between kicks, and the function $f(n)$ will be determined below. The associated classical map is given by

$$
\begin{gathered}
\tilde{p}_{N}=\tilde{p}_{N-1}+\kappa_{1} f(N) \sin \left(\tilde{x}_{N-1}\right)+2 \kappa_{2} \cos \left(2 \tilde{x}_{N-1}\right) ; \\
\tilde{x}_{N}=\tilde{x}_{N-1}+\tilde{p}_{N},
\end{gathered}
$$

where $\tilde{x}=x / x^{0}$ is the scaled dimensionless position variable, $\tilde{p} \equiv p T /\left(m x_{0}\right)$ is the scaled dimensionless momentum vari- 
able, $\kappa_{1}=\lambda_{1} T^{2} /\left(m x_{0}^{2}\right), \kappa_{2}=\lambda_{2} T^{2} /\left(m x_{0}^{2}\right)$, and $\left(\tilde{p}_{N}, \tilde{x}_{N}\right)$ represents the phase space location of a classical trajectory at $\left(N+1-0^{+}\right) T$. The corresponding quantum unitary evolution operator for propagating from time $\left(N-0^{+}\right) T$ to time $(N+1$ $\left.-0^{+}\right) T$ is given by

$$
\hat{F}=\exp \left[i \frac{\tau}{2} \frac{\partial^{2}}{\partial \tilde{x}^{2}}\right] \exp \left[-i f(N) k_{1} \cos (\widetilde{x})\right] \exp \left[i k_{2} \sin (2 \widetilde{x})\right]
$$

with dimensionless parameters $k_{1}=\lambda_{1} T / \hbar, \quad k_{2}=\lambda_{2} T / \hbar, \quad \tau$ $=\hbar T / m x_{0}^{2}$. Let $\hat{\tilde{p}} \equiv \hat{p} T /\left(m x_{0}\right)$, where $\hat{p}=-i \hbar \partial / \partial x$, then one has $[\tilde{x}, \hat{\tilde{p}}]=i \tau$, and $\hat{\tilde{p}}=-i \tau \partial / \partial \tilde{x}$, indicating that $\tau$ plays the role of an effective Planck constant. Note that $\kappa_{1}=k_{1} \tau$ and $\kappa_{2}=k_{2} \tau$.

Clearly, if $\lambda_{2}=0$ and $f(n)=1$ then Eq. (1) reduces to the standard kicked rotor model [14] and the classical map of Eq. (2) becomes the standard map. If $\lambda_{2}=0, f(n)=g(n)$, where $g(n)=1$ for $n=4 j+1,4 j+2$, and $g(n)=-1$ for $n=4 j$ $+3,4 j+4$, where $j$ an integer, then the system becomes that in our previous work $[30,31]$. Our accelerator, introduced here, assumes $\lambda_{2} \neq 0$ and $f(n)=g(n)$. That is, the system is kicked by two fields, one of which reverses the sign of its kicking potential after every two kicks.

Note first the existence of "accelerating trajectories" in the classical delta-kicked dynamics. In particular, for particular values of $\kappa_{1}$, the standard map $\left[\kappa_{2}=0, f(n)=1\right]$ can generate trajectories whose momentum increases (or decreases) linearly with time (at least on the average). These trajectories are a class of transporting trajectories [8]. To see this consider the initial conditions: $\left(\tilde{p}=2 \pi l_{1}, \tilde{x}= \pm \pi / 2\right)$ for $\kappa=2 \pi l_{2}$, where $l_{1}$ and $l_{2}$ are integers. Clearly, these phase space points are shifted by a constant value $\left( \pm 2 \pi l_{2}\right)$ in $\widetilde{p}$ after each iteration, resulting in a quadratic increase of rotational energy. These accelerating trajectories are rather stable insofar as they may persist for values of $\kappa$ close to $2 \pi l_{2}$ (with their average momentum shift after each iteration oscillating around the constant value $\pm 2 \pi l_{2}$ ), thus giving rise to transporting regular islands [8], also called the "accelerator modes" in the standard map case [32,33]. Dramatically, if $f(n)=g(n)$ and $\kappa_{2}=0$, then there can exist much larger transporting islands [30]. These islands are associated with the marginally stable points $\left(\tilde{p}=\left(2 l_{1}+1\right) \pi, \tilde{x}= \pm \pi / 2\right)$ for $\kappa$ $=\left(2 l_{2}+1\right) \pi$. Trajectories on these islands will be shifted by a constant value $\left[ \pm\left(2 l_{2}+1\right) \pi\right]$ in $\tilde{p}$ after each kick.

If classical trajectories are launched from the transporting regular islands, they simply jump to other similar islands located in adjacent phase space cells. For trajectories initially outside the transporting regular islands, the "stickiness" of the boundary between the islands and the chaotic sea induces anomalous diffusion over the phase space. For example, the square of the variance of momentum increases nonlinearly, but not quadratically. This is intrinsically different from the case of normal chaotic diffusion in which the square of the variance of momentum increases linearly with the number of kicks.

As in previous studies on delta-kicked systems, one needs to break the spatial symmetry of the kicking potential to induce ratchet effects. More specifically, our choice of the above model Hamiltonian is motivated by the desire to have large transporting islands in the classical phase space [30] and to break the spatial symmetry of the transporting regular islands. As shown below, the symmetry can be indeed broken by a second kicking field, and yet the remaining transporting islands are still of a significant size and have unidirectional transporting properties. This being the case, the mechanism of the Hamiltonian ratchet effects described below becomes transparent without using previous analyses of the relationship between broken spatial-temporal symmetries and ratchet effects [6,34-37].

\section{CLASSICAL RESULTS}

To demonstrate the feasibility of our basic idea, in this section we present our results using some specific computational examples. Figure 1(a) displays the classical phase space structure of the standard map with $\kappa_{1}=3.8, \kappa_{2}=0$, and $f(n)=1$. The regular islands in Fig. 1(a) are not transporting since the momentum of the trajectories launched from these regular islands is bounded and oscillates periodically. Figure 1(b) shows that if the first kicking field reverses its potential after every two kicks, then the regular nontransporting islands in the standard map are destroyed and new regular islands emerge. A simple computation reveals that those islands seen in Fig. 1(b) are transporting. In particular, classical trajectories launched from regular islands on the left side $(\tilde{x}<\pi)$ of the phase space cell will accelerate, with their $\tilde{p}$ increased by $\approx \pi$ after each kick. Due to the spatial symmetry, these islands always have partners with the opposite transporting property: trajectories launched from those islands on the right side $(\tilde{x}>\pi)$ of the same phase space cell will accelerate in the opposite direction, with their $\tilde{p}$ decreased by about $\pi$ after each kick. These properties of the transporting regular islands seen in Fig. 1(b) indicate that they are associated with the marginal stable points $\tilde{p}=\pi, \tilde{x}$ $= \pm \pi / 2$ in the case of $\kappa_{1}=\pi$. Dramatically, upon introducing the second kicking field in Eq. (2), e.g., $\lambda_{2} / \lambda_{1}=\kappa_{2} / \kappa_{1}$ $=0.2 / 3.8$, the transporting islands no longer appear in pairs as in Fig. 1(b). Rather, as seen in Fig. 1(c), the spatial symmetry of the phase space structures is clearly broken, but the size of the main transporting islands that persist remains significant. Specifically, all the trajectories launched from the regular structures clearly seen in Fig. 1(c) will still accelerate linearly with time; the remaining part of the phase space is exclusively occupied by the chaotic sea. That is, there does not exist similar transporting islands that decreases $\tilde{p}$ in a linear fashion.

For a classical ensemble that uniformly covers the entire phase space cell shown in Fig. 1(c), the spatial and temporal periodicity of the system requires that the average acceleration rate is zero $[7,8]$, i.e.,

$$
a_{c} A_{c}+a_{r} A_{r}=0
$$

where $A_{c}$ and $A_{r}$ denote the areas of the chaotic sea and the regular transporting region, respectively, with their associated average acceleration rate denoted by $a_{c}$ and $a_{r}$. Consider now an initial classical ensemble with $\tilde{p}=0$ and $\tilde{x}$ uniformly 

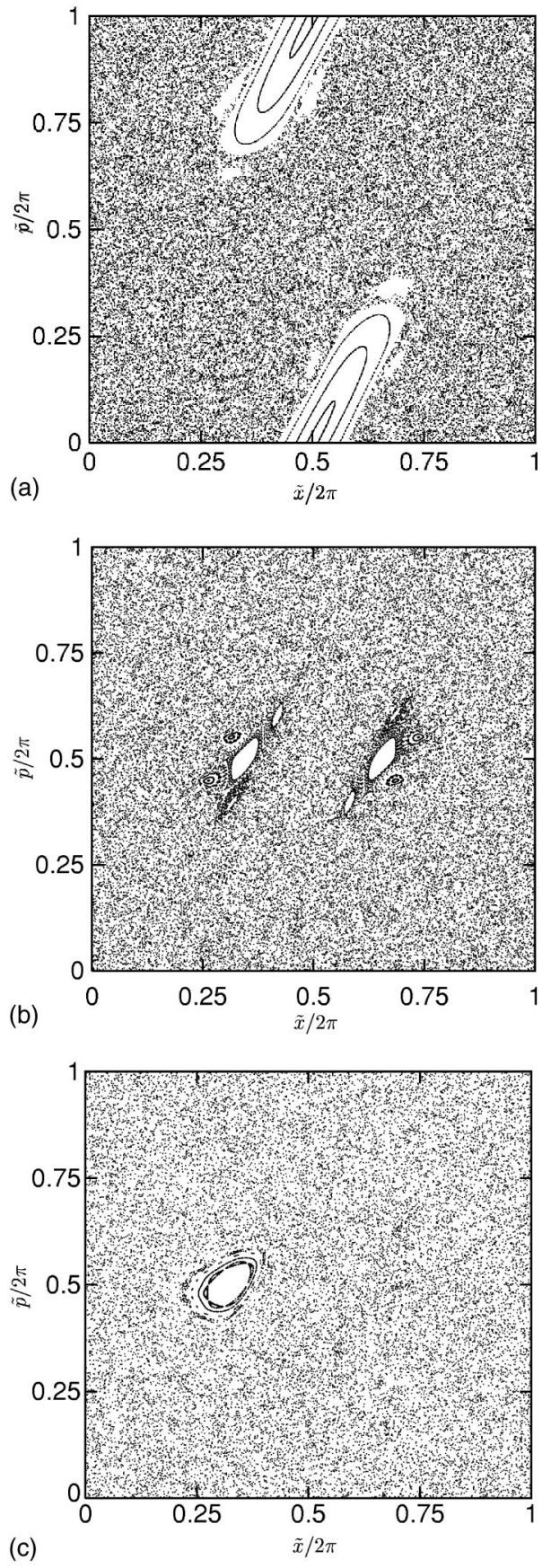

FIG. 1. Classical phase space structures of (a) the standard map $\left[\kappa_{1}=3.8, f(n)=1, \kappa_{2}=0\right]$, (b) a modified kicked rotor model $\left[\kappa_{1}\right.$ $\left.=3.8, f(n)=g(n), \kappa_{2}=0\right]$, and (c) a ratchet accelerator $\left[\kappa_{1}=3.8\right.$, $\left.f(n)=g(n), \kappa_{2}=0.2\right]$. All variables are in dimensionless units. Note that the regular islands seen in (b) and (c) are transporting while those in (a) are not.

distributed between $-\infty$ and $+\infty$. Such an ensemble is easy to simulate both classically and quantum mechanically. Since this initial ensemble is entirely located in the chaotic sea [see Fig. 1(c)], Eq. (4) predicts that the average acceleration rate of this classical ensemble is given by $-a_{r} A_{r} / A_{c}$. This also indicates that the larger the transporting island is, the faster the directed acceleration would be. In the case of Fig. 1(c), $a_{r}=\pi$, so more trajectories travel to the regime of negative $\tilde{x}$.
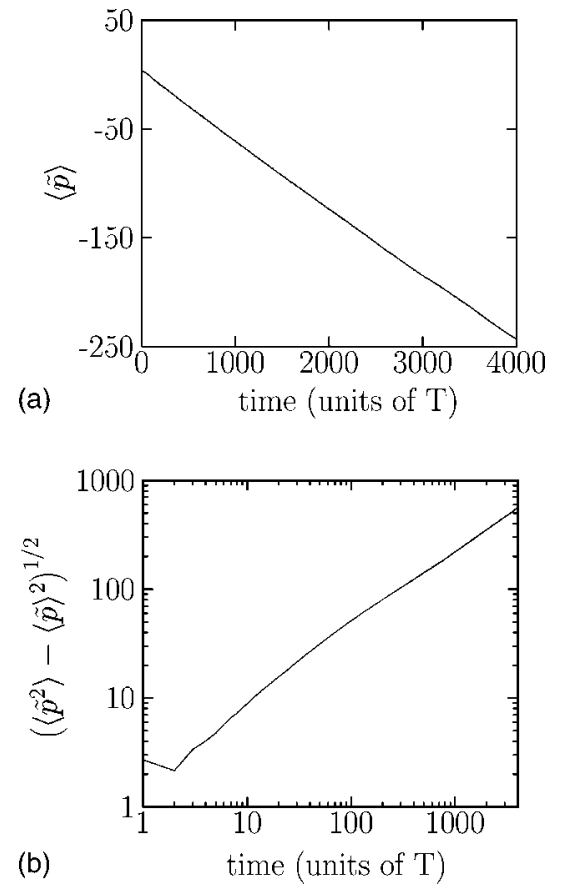

FIG. 2. (a) The linear time dependence of the classical directed current defined as the ensemble averaged value of the momentum, with the initial condition $\tilde{p}=0$ and $\tilde{x}$ uniformly distributed between $-\infty$ and $+\infty$. (b) The associated time dependence of the momentum variance in a log-log plot. The system parameters are the same as those in the case of Fig. 1(c). All variables are in dimensionless units.

That is, even though the average force of the kicking fields is zero and the initial classical ensemble is both spatially symmetric and time-reversal invariant, all the trajectories tend to be accelerated in one direction. Note that, by changing the strength of the second kicking field or varying the relative phase between the two kicking fields [e.g., the second kicking field is replaced by $\lambda_{2} \sin \left(2 x / x_{0}+\beta\right) \sum_{n} \delta(t / T-n)$, where $\beta$ is a relative phase factor], one can also obtain $a_{r}=-\pi$. Hence the acceleration can be also obtained in the reverse direction.

For an initial classical ensemble with $\tilde{p}=0$ and $\tilde{x}$ uniformly distributed from $-\infty$ to $+\infty$, Fig. 2(a) displays the time dependence of the classical net current $\langle\tilde{p}\rangle$, where $\langle\cdot\rangle$ denotes the ensemble average. It is indeed seen that the classical net current is in the negative direction, and accelerates linearly with time. The acceleration rate is also consistent with Eq. (4). Figure 2(b) displays a log-log plot of the time dependence of the associated momentum variance $\Delta_{\tilde{p}} \equiv\left(\left\langle\widetilde{p}^{2}\right\rangle\right.$ $\left.-\langle\tilde{p}\rangle^{2}\right)^{1 / 2}$. A linear fit of the result in Fig. 2(b) gives $\Delta_{\tilde{p}}$ $\sim N^{0.65}$, where $N$ is the number of kicks. This confirms that the diffusion dynamics is anomalous and immediately gives $\Delta_{\tilde{p}} /\langle\tilde{p}\rangle \sim 1 / N^{0.35}$. Hence, as far as the first and second order statistical moments are concerned, the momentum fluctuations of the classical ensemble become less and less important as time increases. In this sense, for sufficiently large times, almost all trajectories in the classical ensemble are accelerated in the negative direction. 


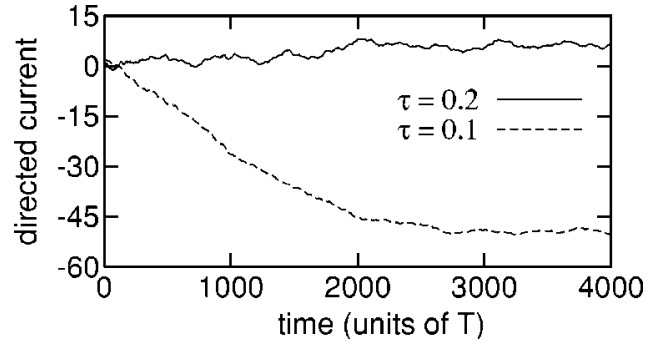

FIG. 3. The time dependence of the quantum dimensionless directed current defined as the expectation value of $\hat{\tilde{p}}$ for two values of the effective Planck constant $\tau$, with the initial condition given by $|0\rangle$ and other parameters are the same as in Fig. 1(c). Results here should be compared with those in Fig. 1(c).

\section{QUANTUM RESULTS}

How then does this ratchet accelerator manifest itself in the corresponding quantum system and how small the effective Planck constant should be in order to observe the analogous ratchet effects in the quantum dynamics? To carry out the quantum calculations we impose a periodic boundary condition on the system, i.e., $|\psi(\tilde{x})\rangle=|\psi(\tilde{x}+2 \pi)\rangle$, where $|\psi\rangle$ is the wave function of the system. This periodic boundary condition suggests that $\tilde{x}$ is now to be understood as an angle variable, and is consistent with the fact that the classical dynamics is invariant if $\tilde{x} \rightarrow \tilde{x}+2 \pi$. Further, our previous work [30] suggests that this choice of boundary conditions should not affect the essence of the quantum dynamics.

Figure 3 shows the net quantum current, i.e., the expectation value of $\hat{\tilde{p}}$ for two values of the effective Planck constant $\tau$, with the initial state given by $|\psi(t=0)\rangle=|0\rangle$. Comparing the results here with that in Fig. 2(a), one sees that the magnitude of the quantum directed current can be much smaller than that of the classical directed current. Interestingly, the directed current in the quantum case displays much more complicated behavior than the linear acceleration seen classically (some slow oscillatory behavior of the quantum current over much longer time scales is also observed, but should be of much less experimental interest). This can be qualitatively explained in terms of a combination of a dynamical localization effect [14] and the quantum tunneling between the chaotic sea and the transporting islands [30,37]. In particular, since the dynamical localization in quantum delta-kicked systems always suppresses the diffusion, the quantum directed current is not expected to grow all the time. More importantly, since the chaotic sea and the transporting islands have opposite acceleration signs [see Eq. (4)], the quantum tunneling between them [30] necessarily decreases the directed current. Indeed, in the case of $\tau=0.2$, one sees that for most of the time the quantum current is in the opposite direction of the classical current. Note that, unlike a previous observation of tunneling-induced current reversal in quantum Brownian motion [38], here the tunneling is between a chaotic sea and a regular region and the system is totally isolated from the environment.

The results in Fig. 3 also suggest that $\tau=0.1$ is small enough to observe the signature of a ratchet accelerator in a quantum $\delta$-kicked system. For example, the quantum current

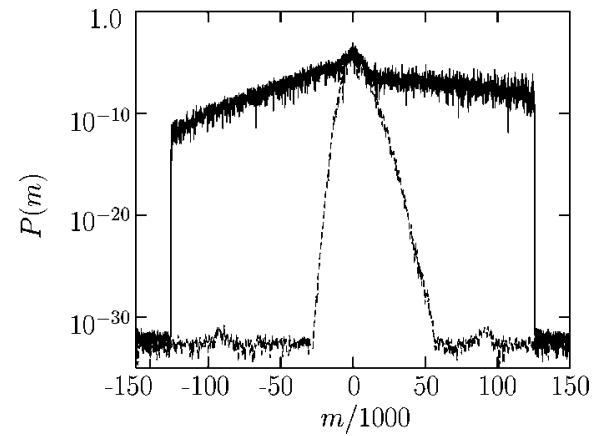

FIG. 4. The probability $P(m)$ of finding the quantum system in state $|m\rangle$ after 4000 kicks, with the initial state given by $|0\rangle$. The solid line is for $\tau=0.1$ and the dashed line is for $\tau=0.2$. Other parameters are the same as those in the case of Fig. 1(c).

for $\tau=0.1$ does increase almost linearly for as many as 2000 kicks, with the average increase rate about three times smaller than in the classical case. This means that for experiments using cold sodium atoms [15] the directed and relatively large quantum current obtained with 2000 kicks would correspond to an average drift velocity on the order of $1 \mathrm{~m} / \mathrm{s}$. Note, however, this value of $\tau$ is about one order of magnitude smaller than what is being examined in atom optics experiments [15], but may be achievable in the future.

It is also interesting to examine the lineshape of dynamical localization with the spatial symmetry of the transporting regular islands broken by the second kicking field. Figure 4 displays the associated distribution function $P(m)$ after 4000 kicks for the same two values of $\tau$. It is clearly seen that for both cases $P(m)$ is asymmetric. Due to the stickiness of the boundary between the transporting islands and the chaotic sea and the associated quantum tunneling, the probability of finding the system in state $|m\rangle$ with a large and positive $m$ can be much higher than that of finding the system in state $|-m\rangle$. This does not contradict the previous observation that after an initial stage the quantum current for $\tau=0.1$ is negative, because the tail of the statistical distribution function $P(m)$ has little effect on the net current, the statistical moment of the lowest order. The huge difference in $P(m)$ between the two cases shown in Fig. 4 with slightly different values of $\tau$ is also noteworthy. This extreme sensitivity of $P(m)$ to the value of $\tau$ is more or less related to the fact that the values of $\tau$ we consider are quite comparable to the area of the main transporting island seen in Fig. 1(c).

\section{CONCLUDING REMARKS}

Using a simple model Hamiltonian system we have demonstrated a new ratchet effect by considering directed classical anomalous diffusion. We have shown that it is possible to achieve directed acceleration with a fixed acceleration rate with two kicking fields that have zero average gradient. This is the case even though the initial classical ensemble is both spatially symmetric and time-reversal invariant. We have also presented some computational examples of the quantum dynamics with different values of the effective Planck constant. It is found that quantum coherence effects may induce 
a reversal of directed current. It is also found that directed quantum current that accelerates linearly with time without using a biased external field should also be achievable in a quantum system that is still far from the classical limit.

We have previously shown that quantum anomalous diffusion in a simple delta-kicked system can be faster than the underlying classical anomalous diffusion [30]. However, the spatial symmetry therein always gives a zero momentum expectation value and therefore one necessarily considers the momentum variance in order to make a meaningful quantum-classical comparison. By contrast, our ratchet accelerator model here allows for a study of the quantumclassical differences in the momentum expectation value itself. As such, the role of the quantum tunneling between a chaotic sea and transporting regular islands becomes even more clear: while it can enhance the increase rate of the momentum variance [30], it suppresses the net acceleration rate of the average momentum in our ratchet accelerator model.

It would be of great interest to consider a molecular realization of the ratchet accelerator, where the variable $x$ is understood as an angle variable and $p$ is understood as an angular momentum variable. Since a microwave field $[18,39]$ will create a kicking potential of the form $\cos (x)$ and an off-resonance laser field $[19,21]$ can create a kicking potential of the form $\cos ^{2}(x)$, a diatomic subject to both microwave and off-resonance laser fields, with their polarization direction perpendicular to one another, is a possible candidate for realizing the ratchet accelerator we propose. However, since in this case the diatomic should be at least described by a three-dimensional rigid rotor, which differs from the planetary kicked rotor in a number of aspects [39], detailed calculations should be carried out to guide the experimental studies. Nevertheless, we believe that this ratchet accelerator model may be of importance for manipulating the rotational motion of diatomics and may provide a means of orienting molecules while accelerating rotation without using a biased external field.

\section{ACKNOWLEDGMENTS}

This work was supported by the U.S. Office of Naval Research, Photonics Research Ontario, and the Natural Sciences and Engineering Research Council of Canada.
[1] P. Reimann, Phys. Rep. 361, 57 (2002).

[2] R. D. Astumian and P. Hänggi, Phys. Today 55 (11), 33 (2002).

[3] P. Jung, J. G. Kissner, and P. Hänggi. Phys. Rev. Lett. 76, 3436 (1996).

[4] J. L. Mateos, Phys. Rev. Lett. 84, 258 (2000).

[5] M. Porto, M. Urbakh, and J. Klafter, Phys. Rev. Lett. 85, 491 (2000).

[6] S. Flach, O. Yevtushenko, and Y. Zolotaryuk, Phys. Rev. Lett. 84, 2358 (2000).

[7] T. Dittrich, R. Ketzmerick, M. F. Otto, and H. Schanz, Ann. Phys. (Leipzig) 9, 755 (2000).

[8] H. Schanz, M. F. Otto, R. Ketzmerick, and T. Dittrich, Phys. Rev. Lett. 87, 070601 (2001). Transporting trajectories can be transporting in either momentum or position, whereas accelerating trajectories are transporting in momentum and hence accelerate.

[9] T. Cheon, P. Exner, and P. Seba, J. Phys. Soc. Jpn. 72, 1087 (2003).

[10] S. Denisov, J. Klafter, and M. Urbakh, Phys. Rev. E 66, 046203 (2002).

[11] T. S. Monteiro, P. A. Dando, N. A. C. Hutchings, and M. R. Isherwood, Phys. Rev. Lett. 89, 194102 (2002).

[12] T. Jonckheere, M. R. Isherwood, and T. S. Monteiro, Phys. Rev. Lett. 91, 253003 (2003).

[13] P. H. Jones, M. Goonasekera, H. E. Saunders-Singer, and D. R. Meacher, quant-ph/0309149(2003).

[14] G. Casati and B. Chirikov, Quantum Chaos: Between Order and Disorder (Cambridge University Press, New York, 1995).

[15] F. L. Moore, J. C. Robinson, C. F. Bharucha, B. Sundaram, and M. G. Raizen, Phys. Rev. Lett. 75, 4598 (1995); H. Ammann, R. Gray, I. Shvarchuck, and N. Christensen, ibid. 80, 4111 (1998); J. Ringot, P. Szriftgiser, J. C. Garreau, and D. Delande, ibid. 85, 2741 (2000); M. B. d'Arcy, R. M. Godun, M. K. Oberthaler, D. Cassettari, and G. S. Summy, ibid. 87, 074102 (2001).

[16] S. Fishman, D. R. Grempel, and R. E. Prange, Phys. Rev. Lett. 49, 509 (1982).

[17] G. Benenti, G. Casati, I. Guarneri, and M. Terraneo, Phys. Rev. Lett. 87, 014101 (2001).

[18] R. Blümel, S. Fishman, and U. Smilansky, J. Chem. Phys. 84, 2604 (1986).

[19] I. Sh. Averbukh and R. Arvieu, Phys. Rev. Lett. 87, 163601 (2001).

[20] M. Machholm and N. E. Henriksen, Phys. Rev. Lett. 87, 193001 (2001).

[21] M. Leibscher, I. Sh. Averbukh, and H. Rabitz, Phys. Rev. Lett. 90, 213001 (2003).

[22] P. Facchi, S. Pascazio, and A. Scardicchio, Phys. Rev. Lett. 83, 61 (1999).

[23] B. Georgeot and D. L. Shepelyansky, Phys. Rev. Lett. 86, 2890 (2001).

[24] G. Kurizki, M. Shapiro, and P. Brumer, Phys. Rev. B 39, 3435 (1989); M. Shapiro and P. Brumer, Principles of the Quantum Control of Molecular Processes (John Wiley, New York, 2003).

[25] R. Atanasov, A. Hache, J. L. P. Hughes, H. M. van Driel, and J. E. Sipe, Phys. Rev. Lett. 76, 1703 (1996).

[26] R. D. R. Bhat and J. E. Sipe, Phys. Rev. Lett. 85, 5432 (2000).

[27] E. Dupont, P. B. Corkum, H. C. Liu, M. Buchanan, and Z. R. Wasilewski, Phys. Rev. Lett. 74, 3596 (1995).

[28] A. Hache, Y. Kostoulas, R. Atanasov, J. L.P. Hughes, J. E. Sipe, and H. M. van Driel, Phys. Rev. Lett. 78, 306 (1997).

[29] M. J. Stevens, A. L. Smirl, R. D. R. Bhat, A. Najmaie, J. E. Sipe, and H. M. van Driel, Phys. Rev. Lett. 90, 136603 (2003).

[30] J. Gong, H. J. Wörner, and P. Brumer, Phys. Rev. E 68, 
026209 (2003).

[31] J. Gong, H. J. Wörner, and P. Brumer, Phys. Rev. E 68, 056202 (2003).

[32] A. Iomin, S. Fishman, and G. M. Zaslavsky, Phys. Rev. E 65, 036215 (2002).

[33] R. Roncaglia, L. Bonci, B. J. West, and P. Grigolini, Phys. Rev. E 51, 5524 (1995).

[34] S. Denisov and S. Flach, Phys. Rev. E 64, 056236 (2001).

[35] S. Denisov, J. Klafter, M. Urbakh, and S. Flach, Physica D 170, 131 (2002).

[36] S. Denisov, S. Flach, A. A. Ovchinnikov, O. Yevtushenko, and
Y. Zolotaryuk, Phys. Rev. E 66, 041104 (2002).

[37] The quantum tunneling between the chaotic sea and the transporting island implies that the Floquet state of the kicked quantum system can simultaneously occupy these classical phase space structures, a counterintuitive result first demonstrated in L. Hufnagel, R. Ketzmerick, M. F. Otto, and H. Schanz, Phys. Rev. Lett. 89, 154101 (2002).

[38] P. Reimann, M. Grifoni, and P. Hänggi, Phys. Rev. Lett. 79, 10 (1997).

[39] J. Gong and P. Brumer, J. Chem. Phys. 115, 3590 (2001). 